\title{
Una aproximación a las modalidades y elementos de las ayudas públicas anticompetitivas ${ }^{1}$
}

\section{An approach to the modalities and elements of anti-competitive public aids}

Recibido: 02 de diciembre de 2015 - Revisado: 20 de mayo de 2016 - Aceptado: 24 de junio de 2016.

Jesús Alfonso Soto Pineda ${ }^{2}$

\section{Resumen}

La defensa de la libre competencia configura una de las herramientas más importantes en el entorno global y un elemento fundamental de la política económica, centrado en controlar las conductas capaces de restringir la competencia propiamente dicha y el acceso al mercado. Así, en entornos de gran relevancia económica, el control de ayudas públicas se ha instaurado como uno de los elementos fundamentales de esta política. Razón por la cual en el presente artículo se abordará la implementación de dicho control en entornos en los cuales aún no es una realidad. Para ello, se presentan dos criterios que determinan la legalidad o ilegalidad concurrencial de una ayuda pública, sus modalidades; y los elementos que deben converger en estos subsidios para que resulten anticompetitivos.

\section{Palabras clave}

Defensa de la libre competencia, ayudas públicas, subsidios, modalidades, elementos, configuración, efectos anticompetitivos.

\begin{abstract}
The defense of free competition is one of the most important tools in the global environment and a fundamental element of economic policy, focused on controlling behaviours capable of restricting competition and access to the market. Thus, in environments of great economic importance, the control of public aid has been established as one of the fundamental elements of this policy. This is the reason why this article will address the implementation of such control in environments where it is not yet a reality. To do this, two criteria are presented that determine the legality or concurrent illegality of a public aid, its modalities; and the elements that must converge in these subsidies to be anti-competitive.
\end{abstract}

\section{Keywords}

Defense of free competence, public aid, subsidies, modalities, elements, configuration, anti-competitive effects.
${ }^{1}$ El presente artículo de investigación forma parte del proyecto "La Defensa de la competencia", que adelanta el autor como miembro del Grupo de Investigación en Derecho y Economía del Departamento de Derecho Económico de la Universidad Externado de Colombia.

2 Profesor investigador en Derecho de la competencia y el consumo del Departamento de Derecho Económico de la Universidad Externado de Colombia. Abogado de la Universidad Externado de Colombia. Magíster en Derecho Empresarial de la Universidad Autónoma de Madrid. Doctor en Derecho y Ciencia Política por la Universidad Autónoma de Madrid.

Correo electrónico: jesus.soto@uexternado.edu.co

Para citar este artículo use: Soto, J. (2017). Una aproximación a las modalidades y elementos de las ayudas públicas anticompetitivas. Civilizar Ciencias Sociales y Humanas, 17(32), 27-48. Doi: 10.22518/16578953.816 


\section{Introducción}

En el marco de protección de la libre competencia en Colombia y en los países del entorno resulta común que -al margen de la naturaleza de los actores involucrados- las conductas con capacidad para deformar el entorno competitivo sean siempre enmarcadas en una de las dos conductas "tipo" que componen el ideario principal de la defensa de los mercados: la colusión y el abuso de posición de dominio. El concepto de "colusión pública" es un ejemplo de ello, toda vez que configura el argumento por excelencia que le es aplicable en el entorno mencionado a los comportamientos anticoncurrenciales en los cuales alguno de los componentes es público, pese a que la experiencia comparada ofrece calificaciones diversas y de mayor precisión, que atienden principalmente a la naturaleza y condiciones de la implicación del sujeto público en la conducta.

Así las cosas, si bien la colusión pública es una realidad en el ámbito de la defensa de la competencia, dicha denominación no puede hacerse extensiva a todos aquellos casos en los cuales un agente de naturaleza pública sea participe en la conducta anticompetitiva. La colusión pública alude, principalmente, a acuerdos entre licitadores dirigidos a falsear las condiciones del concurso mediante la presentación de propuestas concertadas al alza, dirigidas a que el ganador obtenga las mejores condiciones por parte del sector público y compense con dicha ganancia a los demás licitadores miembros del pacto. En aquella condición no están adheridas, por tanto, otras conductas que también involucran el sector público, en virtud de las cuales los Estados, instituciones, órganos y/o poderes públicos, toman la decisión de favorecer a ciertas empresas de su entorno por medio del otorgamiento de ayudas públicas ${ }^{1}$.

El favorecimiento mediante una ayuda pública no hace referencia a una colusión pública $^{2}$. Aunque no por ello comporta efectos de mayor levedad para el ecosistema competitivo. Por el contrario, las evidencias advierten que las ayudas públicas pueden significar la conducta de mayor riesgo para la estabilidad concurrencial (García \& Neven, 2005; Mollgaard, 2007) al comportar una intromisión pública en el desempeño privado. El otorgamiento por parte de autoridades públicas de auxilios a algunos participantes del mercado que no han logrado crear de forma autónoma las eficiencias correlativas a su posición competitiva (Soto, $2014 \mathrm{a}$, p. 321), repercute negativamente en las condiciones del sector económico implicado, afectando de forma directa o transversal a los competidores no receptores de dicha asistencia (Estoa, 2006; Pérez, 2008).

Resulta común que los Estados, administraciones regionales, sectoriales y municipales, así como circunscripciones análogas, sean proclives a favorecer a las empresas locales (Meiklejohn, 1999, p. 7). Razón por la cual desde varios sistemas alrededor del globo se ha estructurado una respuesta dirigida a impedir que los mencionados subsidios desestabilicen los mercados y falseen la competencia (Lin \& Milhauptl, 2013, p. 811; Soto, 2014b, pp. 1529). El caso de mayor relevancia y alcance es el de la Unión Europea, donde se han dado pasos contundentes hacia el control de ayudas públicas al interpretar que la estructura plural y supranacional que compone la idea comunitaria en el viejo continente alberga mayores riesgos de restricción competitiva mediante auxilios, ante la variedad de elementos y agentes públicos y privados que participan en el Mercado Común Europeo (Collie, 2000, p. 867; Martin \& Valbonesi, 2006, p. 136). Siendo por tanto la principal fuente de consulta acerca del alcance de la conducta y del recorrido de la misma desde la perspectiva de las fuentes del Derecho.

En otros sistemas de relevancia como el de los Estados Unidos, los subsidios también han gozado de cierta consideración en el marco de la Defensa de la competencia. Siendo los Tribunales estatales y federales del país nor- 
teamericano los que han impulsado en mayor medida el crecimiento del antitrust -en virtud del alto nivel de litigiosidad de la materia en dicho país-, han sido precisamente aquellos quienes han generado mediante algunos de sus fallos un impulso en la "gestión" de la conducta (Schinkel, 2007, p. 539), al entenderla además de discriminatoria y nociva para los intercambios comerciales entre los Estados miembros de la Unión federal, contraria a la Commerce Clause en la cual se soporta en gran medida el sistema económico estadounidense (Wood, 2007). Los precedentes de creación de leyes, puesta en marcha de políticas de planificación industrial en favor de las compañías locales y la competitividad desmedida entre los Estados dirigida a lograr inversión mediante la concesión de subsidios, han generado que las ayudas públicas sean un tema cada vez más recurrente en el país norteamericano, que demanda restricciones, controles y/o prohibiciones taxativas (Organización para la Cooperación y el Desarrollo Económicos -OCDE, 2010, pp. 3-4).

Otros sistemas relevantes de Defensa de la competencia han hecho eco de la regulación y control implementado en la Unión Europea y de la importancia "tácita" de la cual goza en Estados Unidos. Países como Canadá y Australia han dado pasos hacia la implementación de un sistema que prevenga al mercado interno de los subsidios de las autoridades territoriales del país, así como también de terceros países. Brasil se ha erigido como uno de los mayores defensores del control de subsidios a escala global (Moori, 2007), en especial aplicación del acuerdo sobre subvenciones y medidas compensatorias adoptado tras la Ronda de Uruguay por los países miembros de la Organización Mundial del Comercio, pues en su opinión los efectos nocivos generados por un Estado mediante el otorgamiento de ayudas públicas no se limitan a los mercados domésticos (Alonso, 2001) ${ }^{3}$ donde surgieron ${ }^{4}$, sino que también se extienden hacia el exterior, impactando las economías de los socios comerciales del país otorgante de las ayudas (Calvo \& Carrascosa, 2001, p. 223;
Quereb, 2002, p. 387). Japón y Corea del Sur han implementado regímenes estrictos sobre los subsidios en lo concerniente a sus relaciones comerciales con la Unión Europea; y poco a poco las ayudas públicas se han extendido como una conducta riesgosa en términos concurrenciales, que amerita una regulación e implementación institucional adecuada.

Hispanoamérica es otro ejemplo de la creciente importancia de la conducta en el ámbito de la Defensa de la competencia. Su doctrina ha incrementado escalonadamente el interés por la conducta y ha iniciado el camino hacia la definición de sus criterios principales, tanto en lo alusivo a las relaciones comerciales entre Estados como a lo referido a las relaciones entre circunscripciones locales al interior de un Estado (Whish \& Bailey, 2012). En Colombia se han generado ecos en aquella dirección y se han sugerido controles basados en aplicar la normativa en vigor ${ }^{5} \mathrm{o}$ en crear un nuevo marco regulatorio de Defensa de la competencia que otorgue respuestas a los extremos sustanciales y procesales de la conducta (Soto, 2014a).

En ese sentido, dado el progresivo impacto del componente concurrencial en mención y la vocación inequívoca que tienen las ayudas públicas para generar impactos restrictivos en los mercados hispanoamericanos -y de forma concreta en el colombiano-, resulta esencial preguntarse ¿cuáles son los extremos que influyen en la consideración de los subsidios, como legales y acordes con la protección de la libre competencia, o como ilegales e incompatibles con dicho ideario? Identificar las modalidades de ayuda pública que en el marco comparado generalmente han presentado contradicciones con la Defensa de los mercados; así como también definir los elementos que permiten establecer una ayuda como ilegal, resulta más que esencial para responder aquel cuestionamiento y de tal modo sentar las bases de una hipotética implementación de un control de esta conducta en Colombia y en los países del entorno. 
Como consecuencia a ese imperativo, el presente artículo de investigación, que es a su vez un avance del proyecto sobre ayudas públicas que adelanta el autor, realizará una exposición de aquellos extremos primarios que influencian el alcance de una ayuda pública incompatible con la defensa del ecosistema competitivo, agrupando la experiencia comparada -con especial atención a la Unión Europea por su experiencia extendida- en materia normativa, doctrinal y jurisprudencial. Por lo tanto, en la primera parte del documento, además de realizar una calificación conceptual sucinta de las ayudas públicas en el marco de la protección de la libre competencia, se realizará una exposición de las modalidades de auxilio público -clásicas y novedosas-en las cuales se encubre la intención de favorecer los intereses de la empresa local frente a la foránea. A renglón seguido, en la segunda parte del artículo, se realizará una descripción de los elementos que debe adherir una ayuda pública para ser considerada incompatible -independientemente del sistema y emplazamiento involucradocon el ideario de la protección de la libre competencia, de acuerdo también a la unificación de las experiencias comparadas aunque nutriéndose del mayor recorrido de la Unión Europea- y a los procesos resultado de la aplicación de fuentes, que se han suscitado en diversos sistemas.

Mediante la exposición de aquellos criterios esenciales que deben ser tomados en cuenta por la autoridad de competencia -o encargada equivalente- para realizar una valoración de los supuestos de hecho relacionados, la investigación que ha dado lugar al artículo cumplirá dos objetivos concretos. Así pues, en primera medida, evidenciará la peligrosidad concurrencial de los auxilios públicos exhibiendo sus modalidades naturales en el entorno global -las cuales a su vez responden a conductas cotidianas y normalizadas en la realidad de las interacciones del poder público y el sector empresarialrealizando una sugerencia de implementación del control de subsidios. En la misma dirección y en segunda medida, el artículo favorecerá la evaluación de ayudas públicas futuras, toda vez que el marco sustancial del control se encontrará de inicio más nutrido, simplificando la tarea de estudio y examen de la autoridad encargada de liderar la implementación de un sistema que ya resulta imperativo para la evolución de la Protección de la libre competencia.

\section{Ayudas públicas anticoncurrenciales y sus modalidades}

En el ámbito de la Defensa de la competencia, las ayudas públicas pueden ser definidas como ventajas selectivas que se confieren con cargo a fondos públicos a actores de los diversos sectores productivos de la economía. Las ayudas públicas, también desarrolladas en el marco doctrinal como ayudas de Estado, subsidios, auxilios públicos, asistencias, apoyos, etc., configuran una conducta con un gran potencial de distorsión del ecosistema competitivo y por ello ameritan un control exhaustivo usualmente liderado por la autoridad de competencia del sistema de referencia (Calvo \& Carrascosa, 2001; Querub, 2002).

Por el peligro que representan y con el ánimo de garantizar que la labor de rastreo y descubrimiento de los auxilios públicos implica un mayor esfuerzo para la autoridad de competencia - tanto en los sistemas en los cuales configuran una conducta prohibida como en aquellos en los que actualmente se presenta un interés escalonado por controlarlas- las entidades públicas y las empresas favorecidas con este tipo de ayudas, de forma colegiada, suelen modificar y combinar los mecanismos de auxilio tradicionales creando sistemas alternos de apoyo, inmensamente complejos (Bacon, 2004, p. 337), en los cuales resulta difícil identificar los instrumentos de hecho y de derecho puestos en marcha para consolidar el apoyo (Comisión Nacional de Competencia, 2008, p. 13). El incremento de modalidades 
resultante de aquella dinámica hace de las ayudas públicas una herramienta de creciente peligro para falsear la competencia, toda vez que gracias al ejercicio farragoso de las acciones públicas y privadas de Defensa de la competencia, múltiples ventajas concedidas pueden devenir indemnes.

Más allá de aquellos esfuerzos realizados por los agentes intervinientes, los auxilios responden a unas formas clásicas recurrentes en las cuales pueden encuadrarse, en su mayoría, los auxilios otorgados por los poderes públicos ${ }^{6}$. Lo cual bajo ningún concepto significa que estén restringidas a unas pocas las modalidades de auxilio ilegal, toda vez que en los instrumentos jurídicos que sirven de referencia al control de este tipo de ayudas, resulta indiferente la forma que pueda ser utilizada por los Estados y las autoridades análogas para apoyar a sus empresas, ya que se ha entendido que la delimitación restrictiva de los sistemas de ayuda pública puede reprimir la evolución progresiva del concepto de auxilio, facultando al "poder público", de forma trasversal, a coadyuvar el ejercicio económico privado (Hovenkamp, 1999).

Circunstancia que no ha impedido que la jurisprudencia, la doctrina internacional especialmente la europea- y las autoridades de competencia que hacen las veces de supervisoras de este tipo de conductas, hayan relacionado diversas modalidades frecuentes que dan lugar a los auxilios públicos, dejando clara, en todos los casos, la intención meramente expositiva del listado y su relatividad (Steenkolenmijnem vs. La Alta Autoridad de la CECA, 1961, febrero 23). Las formas de ayuda se encuentran en una evolución continua hacia mecanismos de inmensa complejidad que hacen estéril cualquier esfuerzo por estructurar un listado taxativo inalterable de sistemas de apoyo público. Siendo todas aquellas consideraciones la razón por la cual se entiende imperativo exponer a renglón seguido, las modalidades globales de mayor "popularidad".

\section{La transferencia patrimonial.}

Como bien se extrae de su rótulo, esta modalidad de ayuda pública se basa en realizar una atribución patrimonial directa con cargo a fondos públicos y en favor de una o varias empresas determinadas, dirigida a otorgar una ventaja competitiva que en estricta atención de las condiciones habituales del mercado no hubiese sido posible obtener (Bercovitz, 1986, p. 463; Rodríguez, J., 2002, p. 53).

En esta modalidad se encuentran las atribuciones patrimoniales que son realizadas por el poder público a favor de las empresas sin retorno suficiente ni justificación adecuadatanto en dinero como en especie. Estando así adheridas múltiples configuraciones que han dado lugar a variados supuestos de hecho en el entorno económico global, y especialmente en el viejo continente.

Dentro de las formas más empleadas por los Estados y administraciones, además de la "cesión de liquidez" que configura la subvención por excelencia mediante la cual se pone en práctica la trasferencia patrimonial ${ }^{7}$-en virtud del gran número de casos que genera y la simplicidad de ejecución que comporta ${ }^{8}$-, es posible identificar algunos otros instrumentos que han sido empleados de forma recurrente por el "poder público" en el marco de la Defensa de la competencia a escala global.

Así, dentro de los instrumentos de ayuda que encajan en esta modalidad resaltan (i) la venta de propiedades públicas $\mathrm{y} / \mathrm{o}$ activos a agentes privados a precios inferiores a los del mercado; (ii) la prestación de servicios por parte de la administración a título gratuito o a precios por debajo de los imperantes ${ }^{9}$; (iii) la compra de propiedades empresariales $\mathrm{y} / \mathrm{o}$ activos privados a precios elevados por encima de los valores del mercado (Tasan-Kok, Groetelaers, Haffner, Van der Heijden \& Korthals, 2013, pp. 628642); (iv) el consumo reiterado por parte de la administración de bienes o servicios de una 
empresa frente a los de otras competidoras (Sentencia del Tribunal de Primera Instancia de la Unión Europea [STPI], T-14/96.6 Bretagne Angleterre Irlande vs. La Comisión de las Comunidades Europeas); así como también (v) la construcción de infraestructuras por parte de la administración con el único objetivo de beneficiar a empresas determinadas o a industrias privadas concretas elegidas de forma selectiva ${ }^{10}$.

\section{Los Auxilios fiscales.}

Las medidas de carácter fiscal implementadas por los Estados, administraciones territoriales y circunscripciones análogas, forman parte del catálogo de conductas tácitas susceptibles de colisionar con un régimen de control de ayudas estatales adecuadamente implementado. La capacidad que atesoran los instrumentos tributarios para reducir la carga presupuestaria de las empresas, así como para generar beneficios y/o ventajas competitivas, hacen de esta forma de apoyo el epicentro de los esfuerzos que deben realizar las autoridades a cargo del control de ayudas públicas en el marco concurrencial (Martín, 2012, p. 38).

Aunque comportan un peligro indubitado para el ecosistema competitivo, no es posible hallar disposiciones taxativas sobre ellas en los instrumentos normativos que hasta hoy soportan el engranaje del control en el marco del comercio internacional o en sistemas como el de la Unión Europea o el de los Estados Unidos. En el ámbito comunitario el artículo 107 del Tratado de Funcionamiento de la Unión Europea (TFUE), así como las normas precedentes que regulaban dichos auxilios en el Tratado Constitutivo de la Comunidad Europea (TCCE) y el Tratado Constitutivo de la Comunidad Europea del Carbón y delAcero (Tratado CECA), se inclinaron por la "no adhesión" de listados o condiciones restrictivas acerca de los instrumentos de apoyo público prohibidos en el espacio económico europeo, pues se interpretó que las disposiciones abstractas y generales podrían favorecer en mayor medida la evolución conceptual de las ayudas públicas, implementando instrumentos adecuados de interpretación (Soto, 2014c, p. 360). Criterio que parece haber coincidido en la estructuración del Acuerdo sobre Subvenciones y Medidas Compensatorias de la Organización Mundial del Comercio, pues más allá de alguna referencia escueta en el artículo 25.3, la norma elige realizar planteamientos generales que favorecen la aplicación a supuestos de hecho concretos (García, 2012, p. 98).

El silencio de la norma no implica, por tanto, que no sean de relevancia las exenciones fiscales, los incentivos tributarios, las deducciones o las normas tributarias que generan efectos favorecedores en las empresas, sino, por el contrario, que el control de ayudas públicas requiere de una implicación total del plano institucional, doctrinal (Martín, 2008, p. 900) y jurisprudencial, que permita habilitar formulas efectivas para contener el alcance de todas aquellas medidas, incluidas las tributarias, que además de mitigar -así sea de forma parcial- algunas cargas propias de la actividad empresarial, tienen capacidad para afectar el tránsito económico en el nivel doméstico y supranacional.

En la Unión Europea el tema ha formado parte del ideario principal de las relaciones económicas entre Estados miembros. En los últimos 60 años el debate acerca de las conductas fiscales nocivas ha formado parte del día a día en la Comunidad ${ }^{11}$. En 1996, por ejemplo, como parte de esa intención, surgió uno de los instrumentos de mayor impacto al otorgarle una atención especial al tema: la Comunicación relativa al Código de Conducta en materia de Fiscalidad de las empresas ${ }^{12}$, dirigida a excluir por medio de un compromiso de diversas índoles todas las medidas fiscales de naturaleza perniciosa (Cayero, 2006, p. 83) que descompensasen el comercio interestatal.

No siendo ni mucho menos el único recurso que le ha dado una consideración capital al tema, pues en el camino transitado hasta llegar a la situación actual, en el cual las ayudas 
de Estado -o públicas- otorgadas por medio de medidas fiscales han sido observadas con detalle por las autoridades de competencia en la Unión Europea, previamente surgieron iniciativas que permitieron poner de manifiesto el control imperativo al cual debían ser sometidas (Cayero, 2006, p. 83). Así, por ejemplo, la propuesta que realizó el entonces Comisario de la competencia al Consejo de Asuntos Económicos y Financieros (ECOFIN) en busca de adoptar una política común para combatir métodos desleales de competencia tributaria en los Estados miembros (Comunicación hacia la coordinación fiscal en la UE, 1997). De igual forma la tarea realizada por la Organización para la Cooperación y el Desarrollo Económicos (OCDE) que por medio del conocido documento Harmful Tax Competition. An emerging Global Issue (Avi-Yonah, 2009, pp. 783-795) le otorgó mayor profundidad al debate, convirtiéndolo en una preocupación manifiesta de la Unión Europea (Schon, 1999, p. 912) que sugería, debía ser también una preocupación para otros sistemas faltos del control de ayudas públicas. Sin olvidar la participación del Tribunal de Justicia y la Comisión Europea, que por medio de sus fallos $\mathrm{y}$ resoluciones ${ }^{13}$ han sentado las bases de una prohibición de ayudas estatales de naturaleza fiscal que a día de hoy, comporta pocas dudas.

Conllevando así una obligación actual de los Estados miembros de la Unión Europea y del poder público a nivel doméstico de notificar con carácter previo a la Autoridad de competencia competente, todas aquellas medidas fiscales que pudieran ser consideradas ayudas públicas, con el ánimo de que sean evaluadas, aprobadas, o rechazadas en el seno de la Autoridad, de la mano de los elementos que permiten identificar un auxilio incompatible con el mercado interno, tal y como será expuesto en apartados posteriores.

\section{La participación pública en el capi- tal empresarial.}

Esta modalidad de auxilio es una manifestación habitual de la intervención pública en el tránsito económico. Hace referencia a la inyección de fondos públicos en las empresas a cambio de participaciones o fracciones del capital social. Como forma de apoyo estatal (o análogo), resalta como una de las modalidades de mayor complejidad, dado el gran número de especies particulares de ayudas de mayor singularidad y especialización que se desprenden de ella.

En la práctica este mecanismo (Rodríguez, J., 2002, pp. 59-75) suele ser común, al involucrar procesos de rescate ordinarios, naturales en las administraciones estatales o territoriales, para reflotar y/o socorrer a las empresas, públicas o privadas, que se encuentren en situación de riesgo, en crisis, o inmersas en dificultades económicas de gran profundidad. Debido a la importancia que para el sistema económico nacional o territorial tiene la estabilidad de las empresas de mayor tamaño, estas últimas suelen ser las receptoras habituales de esta modalidad de apoyo. Lo cual llama la atención, pues coincide con una conducta natural de las administraciones a asignar mayores recursos para subvencionar a los agentes de mayor poderío, que aquellos que dispone para favorecer, por ejemplo, a las pymes.

En virtud de aquella realidad, el sistema que otorga mayor atención a las ayudas públicas -el de la Unión Europea- y de forma concreta la máxima autoridad de competencia en aquel sistema, la Comisión Europea, valoran actualmente, con prevención, tras un proceso de evolución continuado ${ }^{14}$, la posibilidad que tiene esta especie particular de ayuda pública para deformar el libre juego del mercado. Razón por la cual ejercen un control atento y minucioso sobre las conductas públicas que pueden encuadrarse en el marco de la prohibición adherida al artículo 107 del TFUE. Tanto la Comisión Europea como el Tribunal de Justicia de la Unión Europea, en sendos fallos, han comprobado aquel extremo ${ }^{15}$. Países como Alemania, el Reino Unido o España ${ }^{16}$, han sido protagonistas de este tipo de ayudas 
en los últimos 15 años acaparando una parte importante de los casos que se han suscitado en torno a la adquisición de participaciones en el viejo continente, creando así, un panorama de efectividad en su control, al configurar una modalidad de largo recorrido sobre la cual la Comisión Europea atesora una experiencia excepcional, que solo hasta ahora empieza a cultivar acerca de otros mecanismos de auxilio de mayor novedad.

\section{La creación de regímenes especia- les de rescate, quiebra y/o reestruc- turación.}

El presente mecanismo de auxilio se configura en aquellos casos en los cuales las administraciones nacionales o territoriales estructuran y ponen en marcha regímenes excepcionales de rescate, reestructuración o quiebra, para favorecer, recuperar y/o reflotar a empresas, que además de contar con un papel protagónico en la realidad económica del emplazamiento representado por la autoridad pública, se encuentren en situación de riesgo al estar inmersas en procesos de naturaleza concursal ${ }^{17}$.

Esta forma de auxilio es común en la actualidad toda vez que ha sido acentuada por las administraciones públicas como respuesta a la crisis económica global que ha impactado de forma profunda tras el año 2008 (Fehling, 2010, pp. 41-64). Su implementación ha sido mayor en el sector financiero, pero también ha tenido cabida en otros sectores fundamentales como el inmobiliario, agrícola, minero, etc. (Almunia, 2001, pp. 1-12).

La implementación de este tipo de políticas de auxilio ha sido flexibilizada en los sistemas en los cuales el control de ayudas públicas es una realidad, al entender que son un medio esencial para superar los desequilibrios surgidos de los periodos de peligro económico. No obstante, en aquellos sistemas también se ha defendido que no pueden convertirse en la regla general, pues debe ser siempre consecuencia natural del mercado que las empresas ineficaces desaparezcan, dejando lugar a los participantes y competidores que han mostrado mayor eficacia en la tarea económica. Razón por la cual en las normas que regulan este tipo de apoyos en la Unión Europea, solo se consideran compatibles los regímenes excepcionales que cumplan de forma simultánea ciertas condiciones estrictas ${ }^{18}$.

En el mismo sentido que otros tipos de auxilio público, la presente modalidad no se encuentra desarrollada de forma taxativa en la normativa de referencia de ayudas públicas. La normativa europea sobre auxilios públicos, las normas internas de los países miembros de la Unión Europea, la normativa estadounidense, los tratados entre Estados que contienen una regulación sobre ayudas, etc., no adhieren una alusión concreta al respecto. En virtud de aquel silencio, algún sector de la doctrina ha interpretado que no configura en sí misma un mecanismo distinto a la transferencia patrimonial pues la inyección de capitales - natural de esta última- suele ser su forma de ejecución más ordinaria. Aun así, están vinculadas con este mecanismo de ayuda pública otras formas de asistencia que también podrían en estricto sentido entenderse como manifestación exclusiva de la primera modalidad expuesta en el presente documento, pero que igualmente son puestas en práctica como parte de sistemas de mayor complejidad como el actual. Verbigracia, la condonación de deuda, el otorgamiento de préstamos con interés reducido, el acceso a garantías peculiares a cargo de la administración para garantizar el acceso al crédito, y las combinaciones surgidas de aquellas modalidades (Fehling, 2010, pp. 43-45).

Sea cual sea la forma utilizada por las administraciones para ayudar a ciertas empresas, lo esencial de esta modalidad de auxilio es la creación de sistemas excepcionales que, vinculados con la dinámica concursal, 
estén a su vez asociados a profundos riesgos económicos (Cárdenas, 2003, p. 115).

\section{Modalidades anexas frecuentes.}

A las formas de subsidio público de mayor recorrido que ya han sido reseñadas, se adhieren otras formas de apoyo estatal de gran novedad que a pesar de no haber provocado una proporción significativa de asuntos, han llamado la atención de las autoridades de competencia empoderadas para ejercer un control sobre los auxilios públicos, en virtud de la capacidad que han comprobado tener para agrupar, de forma simultánea, todos aquellos elementos que hacen de un auxilio particular incompatible con el libre juego del mercado.

En ese sentido, el control de ayudas estatales se extiende actualmente a otros mecanismos de apoyo más particulares, con un componente de innovación y complejidad importante. Entre los cuales se cree conveniente enunciar dos de ellos. Siendo el primero, el de creación de gravámenes extraordinarios en favor de un sector industrial, empresa o grupo de empresas con el objetivo de impulsar su tarea económica (Marcos, 2004, pp. 5-22; Rodríguez, J., 2002, p. 52); y el segundo, el de disminución u otorgamiento de facilidades a una o varias empresas en el pago de obligaciones al Sistema de Seguridad Social (Cárdenas, 2003, p. 84) o en la satisfacción de compromisos de la misma naturaleza ${ }^{19}$.

Casos particulares relacionados con dichas variedades de auxilio han generado múltiples supuestos de hecho, principalmente en la Unión Europea, donde se han generado perjuicios de importancia para las empresas competidoras excluidas del grupo de beneficiarias ${ }^{20}$. Razón por la cual su control ha cobrado protagonismo, toda vez que configuran un peligro real para el ecosistema competitivo al permitir, en los mismos términos que las modalidades de apoyo previamente expuestas, suavizar ciertas cargas presupuestarias propias de la actividad económica.

\section{Los elementos de configuración de una ayuda pública anticoncurrencial}

En la creación de protocolos y estándares para la concreción del control de ayudas públicas que debe ser planteado como prioridad en los sistemas de Defensa de la competencia, resulta esencial definir, además de las modalidades de auxilio que le son comunes al tránsito económico, los ingredientes que de forma conjunta le otorgan al apoyo público recibido por un agente del mercado, la característica de ilegalidad.

La definición de aquellos ingredientes acumulativos no ha sido aún liderada en el entorno regulatorio, pues el acervo normativo de la materia se restringe a las disposiciones surgidas en el seno de la Unión Europea y al acuerdo de la Organización Mundial del Comercio sobre Subvenciones y Medidas Compensatorias. La regulación alusiva a los auxilios no ha abordado de forma suficientemente clara los elementos que debe identificar la autoridad que ejerce el control, los criterios de configuración y los fundamentos que deben valorarse para que dichos elementos se entiendan aplicados a un supuesto de hecho. Esto, a pesar de que todos aquellos componentes son imperativos para definir los méritos de legalidad del apoyo público, o de su ilicitud.

Como resultado de aquella dinámica y como respuesta al carente desarrollo de los elementos de configuración de las ayudas públicas ilegales en el TFUE, la jurisprudencia del Tribunal de Justicia de la Unión Europea ha tomado el testigo estructurando un listado de elementos que permiten configurar el concepto con mayor precisión (Comisión Nacional de Competencia, CNC, 2012, p. 7). La existencia de aquellos requisitos acumulativos ha influenciado la tarea institucional de las autoridades de competencia ${ }^{21}$ en la contención de auxilios estatales incompatibles con el Derecho de la Unión Europea, toda vez que ha brindado herramientas objetivas y estándares 
definidos a aplicar a supuestos de hecho particulares. Del Acuerdo sobre Subvenciones y Medidas Compensatorias adoptado en la Ronda de Uruguay también es posible extraer bases de un catálogo de criterios a valorar por la autoridad competente. Siendo estos criterios y aquellos generados en el seno del máximo Tribunal de la Unión Europea, compatibles. Razón por la cual su exposición, a continuación, se hace imperativa, toda vez que responde al aprovechamiento de la experiencia comparada en la creación de un punto de inicio de mayor evolución ahí donde se estime conveniente implementar un control de ayudas públicas.

\section{Cargo a fondos públicos.}

La existencia de una ayuda pública incompatible con el ecosistema competitivo está condicionada -en virtud de este elemento- a su procedencia, toda vez que para ser considerada como tal dicha ayuda debe responder a una asignación directa o indirecta, que originada en fondos públicos o estatales ${ }^{22}$, esté dirigida a favorecer el ejercicio empresarial o la actividad económica (García, 2012, p. 106).

En ese sentido, para que se configure un auxilio público ilegal, se requiere de una reducción de los ingresos públicos o del patrimonio de la administración ${ }^{23}$ con motivo de un subsidio concreto otorgado por fuera de la lógica del mercado. Lo que, en otras palabras, hace referencia a la indiferencia que ocasiona la forma del auxilio ${ }^{24}$, toda vez que lo fundamental es el efecto del mismo, que debe conllevar una disminución del acervo patrimonial de una autoridad pública ${ }^{25}$.

\section{Selectividad.}

Para que una medida estatal sea considerada a su vez una ayuda pública incompatible con el ecosistema competitivo, dicha medida debe estar dirigida a beneficiar a determinados agentes económicos (Arpio, 2000, p. 132; García,
2012, pp. 101-105), y no a la economía en su conjunto o a un sector industrial al completo. Aquello significa que la medida en cuestión debe suscitarse en virtud del ejercicio de un sistema discriminatorio puesto en marcha por la administración, con arreglo al cual se favorece, por medio de auxilios, a algunos agentes concretos del mercado; dejando por fuera a otros (Martínez \& Ruiz-Almendral, 2006, p. 597; Urrea, 2007, pp. 2-3), que a pesar de encontrarse en una posición análoga ${ }^{26}$, no son receptores de los subsidios (García, 2012, p. 101).

\section{Generación de un beneficio econó- mico.}

Conforme al presente elemento, para ser contrario a Derecho, un auxilio debe suponer una ventaja económica (Böge, 2006, p. 2) para la empresa o empresas receptoras, que aquella(s) no hubiese(n) obtenido en el ejercicio regular de su actividad productiva ${ }^{27}$. Lo que se traduce en el alivio de las cargas financieras y presupuesta$\operatorname{rias}^{28}$ y en la capacidad competitiva excepcional que surge de aquel apoyo (Rodríguez, W., 2002, p. 358).

En ese sentido, están comprendidas en este elemento las prestaciones positivas (transferencias) y todas aquellas formas anexas de apoyo que comporten un paliativo presupuestal para los receptores (Sentencia del Tribunal de Justicia de la Unión Europea, Kimberly Clark, 1996, ap. 34).

\section{Afectación competitiva e impacto en los intercambios comerciales.}

Elemento que se configura en virtud de la capacidad de la ayuda pública para afectar el comercio en el nivel estatal o local (Pérez, 2012 , p. 496). Ingrediente que puede ser comprobado gracias a la demostración de la presencia de la empresa receptora del auxilio -una vez aportados los méritos probatorios que evidencian los elementos precedentes- en los mercados involucrados con el intercambio 
comercial afectado (del nivel doméstico o del nivel internacional $)^{29}$.

El presente elemento responde a una determinación necesaria en entornos de gran tradición comercial como la europea, y en especial en el de la Unión Europea, al poner en riesgo la salud de las transacciones entre Estados miembros (STJUE, España/Comisión, 1994; Cárdenas, 2003, p. 118). De acuerdo con dicha realidad, el simple desequilibrio económico - originado en la adición de un elemento público y externo a la actividad competitiva de un territorio comunitario (STJUE, Costa/Enel, 1964)- es suficiente en la Unión Europea para determinar que la ayuda pública tiene un alcance que va más allá del terreno doméstico donde se engendró (García, 2012, p. 111).

\section{Conclusiones}

El protagonismo que se le ha conferido en el marco de la defensa de la competencia a nivel global a las ayudas públicas, demuestra que la conducta en sí misma se reconoce en dichos entornos como la principal y más perniciosa fuente de distorsión de los ecosistemas competitivos protegidos. La solvencia económica y el acceso a la liquidez que se presumen como caracteres naturales del "poder público", hacen de las subvenciones un instrumento de desestabilización concurrencial más cercano e inminente, que conforme a los precedentes de su acaecimiento, parece merecer de una estructura y procedimientos de respuesta. Tanto la Organización Mundial del Comercio como la OCDE, la Comisión Europea y algunas autoridades análogas de protección de la libre competencia a nivel global, han respondido a aquella realidad y han trabajado en la dirección de implementar un sistema de control que dé respuesta a los efectos nocivos de la conducta.

En el proceso de estructuración e implementación del mencionado control en los sistemas en los cuales aún no es una realidad, resulta imperativo comprender el alcance de dos criterios que influencian la determinación de legalidad o ilegalidad de una ayuda pública. Por lo que, (I) definir las modalidades de apoyo que confiere el poder público con capacidad para deformar el ecosistema competitivo, y (II) concretar los elementos o ingredientes que deben converger en un subsidio para considerarlo ilegal, resulta fundamental.

Así, en lo alusivo al primer criterio, la investigación que ha dado lugar al presente artículo ha identificado que no solo la transferencia patrimonial -desde el poder público hacia la actividad empresarial- conlleva un auxilio "oficial" que puede deformar la aleatoriedad natural de los mercados, toda vez que también aliviar y/o suavizar las cargas presupuestarias de la(s) empresa(s), puede configurar un soporte incompatible con el ideario de la Defensa de la competencia. De tal modo, es posible menoscabar la estabilidad competitiva mediante otras modalidades de apoyo público como la reducción de las obligaciones fiscales, la participación del poder público como accionista en una iniciativa empresarial, el tratamiento especial recibido por una empresa en procesos surgidos de situaciones de insolvencia, los tratos de favor en materia de compromisos con la seguridad social y la creación de gravámenes extraordinarios en beneficio de un sector en concreto.

La casuística demuestra que los supuestos de hecho que han suscitado controversias en el nivel de la Unión Europea -con mayor fortaleza- y en la Organización Mundial del Comercio -con tibios avances surgidos de la ausencia de enforcement-, han escorado y evolucionado hacia mixturas y mecanismos colegiados de gran complejidad que dificultan el rastreo y consecuencia a imponer a las ayudas públicas. Sin embargo, de la investigación se extrae, igualmente, que los mencionados auxilios responden, al ser teorizados, a unas formas usuales que, propensas a combinarse, se complementan convenientemente entre sí.

Por otro lado, en lo referido al segundo criterio, de concreción de los elementos que 
deben converger en un subsidio para evaluar sus méritos de ilegalidad e incompatibilidad con la Defensa de la competencia, de la investigación que ha dado lugar al presente documento se ha logrado extraer que tanto la Organización Mundial del Comercio como la Comisión Europea -por diferentes vías (regulatoria y jurisprudencial)-, han llegado a una conclusión similar acerca de los elementos que deben integrarse en un subsidio para que el mismo sea considerado incompatible con el ideario concurrencial. Aspecto que permite determinar que si bien el alcance de la ayuda concreta es relevante para ejercer su valoración en el seno de la autoridad competente, dicho alcance no modifica, ni condiciona, los ingredientes que permiten evidenciar el impacto negativo que un apoyo público otorgado a una empresa puede conllevar para el mercado en concreto donde se ha originado, al otorgarle a algunos agentes económicos ventajas no obtenidas con arreglo a las eficiencias en el desempeño.

De tal modo, de las fuentes regulatorias y jurisprudenciales mencionadas se extrae que el auxilio público debe, para ser considerado ilegal, (i) ser cargado a fondos públicos $\mathrm{y}$ por tanto responder a una asignación pública dirigida a favorecer el ejercicio empresarial; (ii) ser fruto de una discriminación selectiva que localice el auxilio en determinados agentes económicos, y no en la economía en su conjunto; (iii) generar un beneficio económico en los agentes favorecidos con el subsidio que alivie sus cargas financieras y presupuestarias; así como también que (iv) el auxilio afecte el comercio en los entornos protegidos y menoscabe los intercambios comerciales entre los países, regiones o localidades involucradas.

El control de ayudas públicas en el marco de la defensa de la competencia es una necesidad imperativa que protege a los mercados de las ineficiencias creadas desde el sector público. Definir los criterios sustantivos que soportan el sistema es una necesidad indubitada que merece un ejercicio procedimental que aún no es materia de la presente investigación. La creación de protocolos y estándares para la aplicación de los elementos de configuración de las ayudas públicas ilegales y de la detección de la modalidad de auxilio empleada, si bien es una quimera en el entorno latinoamericano actual, debe liderarse desde la investigación, pues lo contrario creará confusión y aplicación reiterada de conceptos equívocos a circunstancias dispares, tal y como sucede con la llamada colusión pública.

\section{Notas}

${ }^{1}$ En lo sucesivo, "subsidios", “subvencio-
nes", "auxilios", "ayudas" y "apoyos", con sus
complementos "estatal", "de Estado" y “públi-
co", harán referencia a la misma figura.
${ }^{2}$ En Colombia, los casos de mayor relevancia de esta peligrosa conducta, por el contenido jurídico de sus resoluciones, son los que involucraron al grupo Nule y los procesos licitatorios relacionados con la adjudicación de contratos del Instituto Colombiano de Bienestar Familiar, referidos a la operación de las plantas de producción de Bienestarina y la gestión de aspectos varios de los hogares de la mencionada institución. Asuntos resueltos por la Superintendencia de Industria y Comercio, mediante las Resoluciones 54693 del 16 de septiembre de 2013, y 68972 del 25 de noviembre de 2013 (Bienestarina); 54695 del 16 de septiembre de 2013, y 68967 del 25 de noviembre de 2013 (hogares).

3 El autor entiende, al margen del alcance que pueden llegar a tener las ayudas públicas de un Estado en otro, que un proceso como el de globalización económica, no solo está relacionado con las subvenciones en lo referido a la posibilidad de generar un impacto por medio del otorgamiento de un subsidio en otro país o territorio, pues así mismo es posible que en uso de los auxilios públicos un Estado impida, erigiendo barreras de entrada, que las empresas de otros países participen de la 
competencia en el mercado interior en igualdad de condiciones que las empresas "domésticas", haciendo necesaria una protección bilateral o multilateral que elimine aquel riesgo. Dichas actuaciones son, por ejemplo, que (i) se adoptan políticas reguladoras que establecen un marco normativo al cual supeditar la actividad comercial por medio de la disposición y cumplimiento de requisitos; (ii) se delimitan áreas de actividad reservada a favor de empresas nacionales; (iii) se establecen medidas gubernamentales de política comercial, tales como la regulación de las importaciones, el fomento de las exportaciones, el control de cambios, la protección de las pequeñas y medianas empresas, etc.; y (iv) se "permiten" en un entorno de hecho las prácticas contrarias a la libre competencia.

${ }^{4}$ Como por ejemplo en la Unión Europea, enfocada a la implementación de un mercado único interior. $\mathrm{O}$ la de los Estados Unidos, que a pesar de la existencia de una unión, da lugar a la implementación de derroteros e intereses unilaterales emprendidos por los distintos Estados que conforman la República federal.

5 El marco de protección de la libre competencia en Colombia, aludido en la parte inicial del presente documento, se configura mediante la Ley 155 de 1959 (especialmente su artículo 1, que recoge la cláusula de prohibición general), el Decreto 2153 de 1992 y la Ley 1340 de 2009.

${ }^{6}$ Fuentes esenciales de consulta en lo referido a los diversos mecanismos de auxilio: Besley \& Seabrigth (1999); Busom, Martínez y Corchuelo, (2011); Hancher, Ottervanger y Jan (2012); y Mata (1988).

$7 \mathrm{Al}$ respecto, el plan de auxilio que puso en marcha el Estado holandés para recapitalizar la institución bancaria ING, inyectando, por medio de trasferencias de capital, hasta 15 mil millones de euros. En este caso particular, convergieron los elementos que hacen de una ayuda estatal incompatible con el mercado único interior comunitario. Razón por la cual la Comisión Europea entendió que debían reformularse las condiciones del auxilio estatal otorgado, ya que de mantenerse ponían en riesgo el tránsito económico entre Estados miembros, al adherir una total favorabilidad del lado del banco holandés ING. Argumentos que significaron modificaciones en el plan de restructuración del banco y en el retorno periódico del dinero recibido.

8 En efecto, resulta ser un instrumento de menor complejidad que otras modalidades también relacionadas con la trasferencia patrimonial. Como así se demuestra en la Ayuda para I+D a la empresa ITP - Trent 1000 en la cual se indagó en la legalidad de la ayuda otorgada por el Estado español a la empresa mencionada para la investigación y desarrollo de una turbina de baja presión de motor. Ayuda de Estado C 9/2007 (ex N 608/2006) - España. De igual forma, las aportaciones de capital realizadas por el Gobierno austriaco a Head Tyrolia Maresla, IP/95/1443, con decisión del 30 de julio de 1996; la creación por parte de España de un fondo para la adquisición de activos financieros en el año 2008 para reflotar aquel sector. Ayuda de Estado NN 54a/2008 - España; la inversión que el Estado italiano realizó para impulsar la tarea industrial en Cerdeña. Ayuda de Estado N 93/2008 - Italia, entre otros casos.

9 Ejemplo primordial de esta forma de auxilio y su respuesta comunitaria, se configura en la Sentencia del Tribunal Administrativo de Estrasburgo, del 24 de julio de 2003, Societé Brit Air vs. Chambre de Commerce et dindustrie de Strasbourg et du bas-rhin Societe Ryanair, asunto $\mathrm{N}^{\circ}$ 02-04641, en la cual la contrapartida surgida del servicio prestado por un organismo de naturaleza pública no se consideraba suficiente, creando así una ventaja a favor de la empresa demandada. Del mismo modo, la STJUE, del 11 de Julio de 1996, Syndicat français de l'Express international (SFEI) y otros vs. La Poste y otros, asunto C-39/94, apartado 13. 
${ }^{10}$ Esta es una circunstancia común surgida de la intención de las administraciones públicas de seducir a empresas de gran presencia internacional o con connotado poderío económico para que se establezcan en su territorio, bajo la promesa de que la infraestructura que ya ha sido levantada en aquel emplazamiento les permitirá abaratar sus costos y agilizar el proceso de producción. Conllevando para el ámbito territorial implicado una fuente económica y de empleo de gran importancia. Así, merece atención especial en este tipo de apoyo la Comunicación relativa a los elementos de ayuda en las ventas de terrenos $y$ construcciones por parte de los poderes públicos. Ejemplo relevante el que configuran las ayudas públicas a El Pozo alimentación, S. A., Ayuda de Estado C 22/08 (ex N 222/07 y $N$ 242/07). También relevantes la STJUE, del 19 de diciembre de 2012, Mitteldeutsche Flughafen $A G$ y Flughafen Leipzig-Halle GmbH vs. La Comisión de las Comunidades Europeas, asunto C-288/11 P, o del mismo tribunal, del 16 de mayo de 2002, República Francesa vs. la Comisión de las Comunidades Europeas, asunto C-482/99.

${ }^{11}$ Desde los tiempos de vigencia del Tratado CECA, tanto la Comisión como el TJUE pusieron de manifiesto su peligrosidad en uso de los mencionados argumentos. De importancia, el mencionado fallo Steenkolenmijnem vs. La Alta Autoridad de la CECA. Sobre el fallo aludido, Tiebout (1956, pp. 416-424).

${ }^{12}$ Resolución del Consejo y de los representantes de los Gobiernos de los Estados miembros reunidos en el seno del consejo, anexa a las conclusiones del Consejo ECOFIN, del 01 de diciembre de 1997. Publicado en el Diario Oficial de las Comunidades europeas número 2 del 6 de enero de 1998. Análisis de importancia al respecto, realizado por Lamadrid (2005, pp. $1-56)$.

${ }^{13}$ Algunos de ellos, por actualidad. A saber, el MEMO/13/696, del 17 de julio de
2013, por medio del cual la Comisión pide la recuperación de ayudas fiscales concedidas por España a determinadas Agrupaciones de Interés Económico (AIE) y a sus inversores; el asunto SA $37856(2013 / \mathrm{N})$ de ayudas fiscales otorgadas en Italia a la industria petrolera; y el SA 25254 (NN18/2009).

${ }^{14}$ Desde el año 1982 son muchas las resoluciones emitidas por la Comisión, que han decretado como ilegales sistemas de participación estatal en la actividad económica ejercida por los agentes del mercado, tal y como puede comprobarse en Rodríguez, J., (2002, pp. 89-91). Son precedentes de inmensa importancia que permiten deslindar la profundidad de la materia, las decisiones que involucrando al Estado belga configuraron uno de los precedentes principales de aquellos auxilios. A saber, los asuntos Balamundi (Decisión del 10 de marzo de 1982. Publicada en el Diario Oficial de las Comunidades Europeas L 138, del 19 de mayo de 1982); e Intermills (Decisión del 22 de Julio de 1982. Publicada en el Diario Oficial de las Comunidades Europeas L 280, del 02 de octubre de 1982) que fueron entendidos por la máxima autoridad comunitaria como ayudas incompatibles con el ecosistema económico europeo, al otorgar ventajas mediante la toma de participaciones sociales que en el mediano y largo plazo podría perjudicar a otros agentes dedicados a actividades análogas. Del mismo modo, el asunto Leeuwarder (Decisión del 22 de Julio de 1982. Publicada en el Diario Oficial de las Comunidades Europeas L 277, del 29 de septiembre de 1982) que involucró al Estado holandés, al haber invertido en participaciones empresariales de la mencionada empresa, con el ánimo de eludir la situación de riesgo que enfrentaba.

${ }^{15}$ Ejemplo de ello, en el sector automotor europeo, Nicolini, Scarpa y Valbonesi (2012, p. 13); y en el sector naval, Valdés (1992, pp. 1-8).

${ }^{16}$ De resaltar, el auxilio conocido como Estudios Ciudad de la Luz, SA 22668. Decisión del 8 de mayo de 2012. 
${ }^{17} \mathrm{El}$ instrumento comunitario de mayor importancia, la Comunicación de la Comisión Europea, Directrices comunitarias sobre ayudas estatales de salvamento y de reestructuración de empresas en crisis, del 01 de octubre de 2004, publicada en el Diario Oficial de la Unión Europea C 244/2. Igualmente relevantes, en virtud del marco temporal excepcional surgido por la crisis económica y la respuesta de la Unión Europea y sus Estados miembros a manera de ayudas públicas, la Comunicación de la Comisión relativa al Marco temporal comunitario aplicable a las medidas de ayuda estatal para facilitar el acceso a la financiación en el actual contexto de crisis económica y financiera, del 22 de enero de 2009, publicada en el Diario Oficial de la Unión Europea C 16/3; así como también la Comunicación de la Comisión relativa a la aplicación de las normas sobre ayudas estatales a las medidas adoptadas en relación con las instituciones financieras en el contexto de la actual crisis financiera mundial, del 25 de octubre de 2008.

${ }^{18}$ Que hacen referencia a la situación de peligro de la empresa, la necesidad de ser salvada, la excepcionalidad de auxilio, el límite temporal de la ayuda, la restricción del auxilio a una única oportunidad y la intencionalidad del apoyo, toda vez que debe surgir en virtud de una situación de inmenso riesgo, y no con el objetivo de permitir a la empresa favorecida mantener su statu quo en el mercado (Chindooroy, Muller, $\&$ Notaro, 2007, p. 166).

${ }^{19}$ Los auxilios relacionados con el empleo no resultan incompatibles con el mercado interno europeo en todas las oportunidades, toda vez que ciertas ayudas relacionadas con aquella materia se encuentran exentas de la obligación de notificación a las autoridades europeas, de acuerdo con los términos del Reglamento (CE) No. 2204/2002 de la Comisión, del 12 de diciembre de 2002, relativo a la aplicación de los artículos 87 y 88 del Tratado CE a las ayudas estatales para el empleo. No obstante, las ayudas ilegales que se han presentado en la Comunidad suavizando las obligaciones empresariales con los organismos de seguridad social, y sobre las cuales se refiere en exclusiva el presente documento, no se encuentran acogidas por la exención otorgada en el mencionado Reglamento, pues aquel excluye de la obligación de notificación de auxilios solo aquellos apoyos que relacionados con aspectos laborales estén enfocados a la creación de empleo o a la contratación de personas desfavorecidas y en situación de discapacidad física.

${ }^{20}$ En lo referente a ayudas en el pago de obligaciones a la Seguridad Social, el precedente de mayor relevancia puede identificarse en la STJUE, Déménagements-Manutention Transport $S A$, en la cual el Tribunal entendió que las facilidades en el pago de las obligaciones en comento, al ser otorgadas a una sola empresa por parte de la administración, representada por un único organismo, sí constituía una ayuda estatal de las previstas en el artículo 107 del TFUE. De igual modo, en referencia al tránsito de la Comisión en esta modalidad de auxilio, con diversos desenlaces, toda vez que no en todos los casos tras el análisis de la máxima autoridad de competencia comunitaria se ordenó el retorno de los auxilios, el asunto SA 36080 (2013/NN), Italia, Subsidized loans to undertakings that suffered damage caused by the 2012 earthquakes in Emilia Romagna, Lombardy and Veneto for taxes and social security contributions; SA 36861 (2013 NN), Alemania, Reimbursement of social security contributions under short-time work for companies directly affected by the floods of summer 2013; y SA $33606(2011 / \mathrm{N})$, Irlanda, Refund of Social Security Contributions of Seafarers.

${ }^{21}$ Comunitarios y nacionales, ya que estos últimos acompañan la labor de vigilancia y favorecen la dinámica de cooperación al interior de la Red Europea de Autoridades de competencia (Garrido, 2004, p. 154).

${ }^{22}$ Así, sobre este elemento de configuración, el Acuerdo sobre Subvenciones y Medidas 
Compensatorias de la Organización Mundial del Comercio, artículo primero, que define los criterios para considerar que el subsidio es imputable a cargo a fondos públicos, valorando que los apoyos públicos pueden ser directos o transversales, dando opciones para su determinación (i-iv).

${ }^{23}$ Central, territorial, sectorial, etc. STJUE, PreussenElektra $A G \quad v$ Schleswag $A G$ (2001), ap. 58; UTECA v Administración General del Estado (2009), ap. 43; y República Federal Alemana v La CE (1987), ap. 17.

${ }^{24} \mathrm{Y}$ la medida, que puede ser administrativa, reglamentaria, legislativa, etc. (Cárdenas, 2003, p. 115).

${ }^{25}$ Propia también de la exención fiscal, al generar una disminución patrimonial, comprobable en la intención de beneficiar a empresas seleccionadas (Martín, 2012, p. 45).

${ }^{26}$ El criterio de selectividad se denomina en el Acuerdo sobre Subvenciones y Medidas Compensatorias de la Organización Mundial del Comercio, especificidad. Así, el acuerdo mencionado determina en su artículo segundo, además de la somera definición del elemento, un listado de condiciones que permiten definir que el auxilio ha sido limitado a unos pocos sin considerar a todos los agentes que se encuentran en situación análoga.

${ }^{27}$ Ventaja de acuerdo a la jurisprudencia de los EE. UU. Bacchus Imports, Ltd. v Dias, U.S Supreme Court, 468 U.S. 263 (1984) y Oregon Waste Systems, Inc. $v$ Department of Environmental Quality of Oregon, U.S. Supreme Court, 511 U.S. 93, 99 (1994).

${ }^{28}$ El artículo primero, literal b, del Acuerdo sobre Subvenciones y Medidas Compensatorias de la Organización Mundial del Comercio, alude a este criterio y lo define como esencial para considerar una ayuda, incompatible con el ideario del pacto y el ecosistema competitivo de los países participantes. Del mismo modo, el artículo 14 del mismo Acuerdo se refiere a la importancia del beneficio obtenido con el subsidio, como un instrumento esencial para determinar la cuantía del mismo.

${ }^{29}$ Así, por ejemplo, el mencionado Acuerdo sobre Subvenciones y Medidas Compensatorias de la Organización Mundial del Comercio, que en su artículo 6, numeral tercero, se refiere a la imperatividad del elemento "afectación" en la ecuación de valoración del auxilio como ilegal, pues el mismo debe generar un impacto negativo inequívoco que desplace, obstaculice y/o distorsione las condiciones aleatorias del mercado en las cuales se sustenta la economía de mercado y la competitividad.

\section{Referencias}

Almunia, J. (marzo, 2001). Reformas y reestructuración del sistema financiero. Discurso pronunciado en la conferencia " $\mathrm{La}$ reestructuración del sistema financiero" de la Fundación de las Cajas de Ahorros, Madrid, España.

Alonso, R. (2001). Competencia y comercio. El marco general (Documento de trabajo). Recuperado de http://www.uam. es/personal_pdi/derecho/bbagooria/ Hoja\%20Web\%20RJM/RAS\%20-\%20 CompyComer.pdf

Arpio, J. (2000). Las ayudas públicas ante el Derecho europeo de la competencia. Pamplona: Aranzadi.

Avi-Yonah, R. (2009). The OECD Harmful Tax Competition Report: A Retrospective After a Decade. Brookling Journal of International Law, 34(3), 783-795.

Bacon, K. (2004). State Aids in the English Courts: Definition and other Problems. En A. Biondi, P. Eeckhout \& J. Flynn (Eds.), The Law of State Aid in the European 
Union (pp. 337-358). Oxford: Oxford University Press.

Bercovitz, A. (1986). Normas sobre la competencia del TCEE. En E. García de Enterría, J. González \& S. Muñoz, Tratado de Derecho Comunitario Europeo (pp. 329-480). Madrid: Civitas.

Besley, T., \& Seabright, P. (1999). The effects and policy implications of state aids to industry: an economic analysis. Economic policy, 28, 14-17.

Böge, U. (2006). State imposed Restrictions of Competition and Competition Advocacy. En Challenges of Addressing State Imposed or Facilitated Restraints. Discurso pronunciado en el ABA Spring Meeting.

Busom, I., Martínez, E., \& Corchuelo, B. (2011). Obstáculos a la innovación y uso de incentivos ¿subvenciones o estímulos fiscales?. Economía industrial, 382, 35-44.

Calvo, A-L., \& Carrasco, J. (2001). Intervenciones del Estado y libre Competencia en la UE. Madrid: Colex.

Cárdenas, R. (2003). Las ayudas de Estado y el Derecho Comunitario. Madrid: Instituto de Estudios fiscales.

Cayero, J. (2006). Derecho de la competencia en la UE: Ayudas de Estado de carácter fiscal. Zergak. Gaceta tributaria del País Vasco, 32, 63-103.

Chindooroy, R., Muller, P., \& Notaro, G. (2007). Company survival following rescue and reestructuring State aid. European Journal of Law and Economics, 24, 65-186.

Collie, D. (2000). State aid in the European Union: the prohibition of subsidies in the integrated market. International Journal of Industrial Organization, 18(6), 867-884.
Comisión Europea. (1982, mayo 19). Decisión sobre ayudas de Estado. Balamundi. Diario Oficial. L 138. Unión Europea.

Comisión Europea. (1982, septiembre 29). Decisión sobre ayudas de Estado. Leeuwarder. Diario Oficial L 277. Unión Europea.

Comisión Europea. (1982, octubre 2). Decisión sobre ayudas de Estado. Intermills. Diario Oficial L 280. Unión Europea.

Comisión Europea. (1996). Comunicación relativa al código de conducta en materia de fiscalidad de las empresas. Unión Europea.

Comisión Europea. (1996). Decisión de la Comisión Europea. Head Tyrolia Maresla, asunto IP/95/1443. Unión Europea.

Comisión Europea. (1997). Comunicación hacia la coordinación fiscal en la UE. Unión Europea.

Comisión Europea. (1997). Comunicación relativa a los elementos de ayuda en las ventas de terrenos y construcciones por parte de los poderes públicos. Unión Europea.

Comisión Europea. (2002). Reglamento relativo a la aplicación de los artículos 87 y 88 del Tratado CE a las ayudas estatales para el empleo. Reglamento (CE) No. 2204/2002. Unión Europea.

Comisión Europea. (2004). Comunicación sobre directrices comunitarias sobre ayudas estatales de salvamento $y$ de reestructuración de empresas en crisis. Diario Oficial C 244/2. Unión Europea.

Comisión Europea. (2008). Comunicación relativa a la aplicación de las normas sobre ayudas estatales a las medidas adoptadas en relación con las instituciones finan- 
cieras en el contexto de la actual crisis financiera mundial. Unión Europea.

Comisión Europea. (2008). Apertura formal de investigación de la Comisión Europea. Ayuda de Estado C 22/08, asunto (ex N 222/07 y N 242/07) - España. Unión Europea.

Comisión Europea. (2008). Apertura formal de investigación de la Comisión Europea. Ayuda de Estado N 93/2008 - Italia. Unión Europea.

Comisión Europea. (2008). Apertura formal de investigación de la Comisión Europea. Ayuda de Estado NN 54\%/2008 - España. Unión Europea.

Comisión Europea. (2009). Marco temporal comunitario aplicable a las medidas de ayuda estatal para facilitar el acceso a la financiación en el actual contexto de crisis económica y financiera. Diario Oficial C 16/3. Unión Europea.

Comisión Europea. (2011). Apertura formal de investigación de la Comisión Europea. Ayudas fiscales otorgadas en Italia a la industria petrolera, asunto SA 25254 (NN18/2009). Unión Europea.

Comisión Europea. (2011). Decisión sobre ayudas de Estado. Refund of Social Security Contributions of Seafarers, asunto SA 33606 (2011/N) - Irlanda. Unión Europea.

Comisión Europea. (2012). Decisión sobre ayudas de Estado. Estudios Ciudad de la Luz, SA. Unión Europea.

Comisión Europea. (2013). Memo, por medio del cual la Comisión pide la recuperación de ayudas fiscales concedidas por España a determinadas Agrupaciones de Interés Económico (AIE) y a sus inversores. MEMO/13/696. Unión Europea.
Comisión Europea. (2013). Decisión sobre ayudas de Estado. Reimbursement of social security contributions under shorttime work for companies directly affected by the floods of summer 2013, asunto SA 36861 (2013 NN) - Alemania. Unión Europea.

Comisión Europea. (2013). Decisión sobre ayudas de Estado. Subsidized loans to undertakings that suffered damage caused by the 2012 earthquakes in Emilia Romagna, Lombardy and Veneto for taxes and social security contributions, asunto SA 36080 (2013/NN) - Italia. Unión Europea.

Comisión Nacional de Competencia -[CNC]. (2008). Informe anual 2008: Ayudas públicas. España: Autor.

Comisión Nacional de Competencia -[CNC]. (2012). IV Informe anual sobre Ayudas públicas. España: Autor.

Decreto 2153 de 1992. Por el cual se reestructura la Superintendencia de Industria y Comercio y se dictan otras disposiciones. Diario Oficial No. 40.704. Presidencia de la República, diciembre de 1992.

Estoa, A. (2006). El control de las ayudas de Estado. Madrid: Iustel.

Fehling, M. (2010). La reacción de los Estados europeos frente a la crisis y el Derecho europeo de ayudas públicas. Anuario de la Facultad de Derecho de la UAM, 14, 41-64.

García, L. (2012). Las ayudas de Estado en la UE: concepto, requisitos e implicaciones. Dereito, 21(2), 97-127.

García, J., \& Neven, D. (2005). State aid and disortion of Competition: A Benchmark 
Model (HEI, Working paper No. 6). Recuperado de: https://core.ac.uk/download/pdf/6513429.pdf

Garrido, L. (Dir.) (2004). Los Tribunales del orden civil y el Reg. CE 1/2003 del Consejo, de 16/12/02, relativo a la aplicación de las normas sobre competencia previstas en los arts. 81 y 82 del TCCE. En, Defensa de la competencia por los órganos judiciales: El Reg. CE 1/2003 (pp. 1-218). Madrid: Consejo General del Poder Judicial.

Hancher, L., Ottervanger, T., \& Jan, P. (2012). EU State Aids. Londres: Sweet \& Maxwell.

Hovenkamp, H. (1999). Federal Antitrust policy-the law of competition and its practice. St Paul, Minn: West Publishing.

Lamadrid, A. (2005). El código de conducta en materia de fiscalidad de las empresas y su relación con el régimen comunitario de ayudas de Estado. Madrid: Instituto de Estudios Europeos.

Ley 55 de 1959. Por la cual se dictan algunas disposiciones sobre prácticas comerciales restrictivas. Diario Oficial No. 30138. Congreso de la República, diciembre de 1959.

Ley 1340 de 2009. Por medio de la cual se dictan normas en materia de protección de la competencia. Diario Oficial No. 47420. Congreso de la República, julio de 2009.

Lin, L-W., \& Milhaupt, C. (2013). Los grandes grupos empresariales chinos: entendiendo los mecanismos del capitalismo de estado en China. Revista Chilena de Derecho, 40(3), 801-858.

Marcos, F. (2004). El Derecho de la Competencia en los nuevos Estados miembros de la Unión Europea. Gaceta Jurídica de la Unión Europea y de la Competencia, 229, 82-90.
Martín, A. (2008). El régimen jurídico de las ayudas de Estado y las normas de naturaleza tributaria. En N. Carmina (Coord), Convenios fiscales internacionales $y$ fiscalidad de la UE 2008 (pp. 897-908). Bilbao: Wolters Kluwer.

Martín, A. (2012). El concepto de ayuda de Estado y las normas tributarias en materia de imposición directa: estado de la cuestión. Noticias de la Unión Europea, $324,35-50$.

Martin, S., \& Valbonesi, P. (2006). State aid to business. En P. Biachi \& S. Labory (Eds.), International Handbook of industrial policy (pp. 134-152). Amsterdam: Elsevier.

Martínez, R., \& Ruiz-Almendral, V. (2006). Ayudas de Estado selectivas y poder tributario de las CCAA. Revista española de Derecho europeo, 20, 593-640.

Mata, E. (1988). Las ayudas públicas a empresas y la política de defensa de la competencia. Noticias de la Unión Europea, 46, 33-38.

Meiklejohn, R. (1999). State aids and the single market (Reports and studies Series No. 3). Luxembourg: European Commission.

Mollgaard, P. (2007). The competitive effects of state aid in oligopoly (Mimeo Department of Economics, Working paper). Copenhagen. Recuperado de https://www.fep.up.pt/conferences/ earie2005/cd rom/Session\%20VI/VI.H/ Mollgaard.pdf

Moori, L. (2007). A implicacao do direito da concorrencia na defesa comercial. São Paulo: II Premio SEAE.

Nicolini, M., Scarpa, C., \& Valbonesi, P. (2012). State Aid to Business in the European Union: a Focus on the Car Sector (DEM Working Paper Series No. 09-12). Pavia: 
Università Di Pavia. Recuperado de http://economia.unipv.it/docs/dipeco/ quad/ps/DEM/DEMWP0001.pdf

Organización para la Cooperación y el Desarrollo Económicos -[OCDE]. (2010). Global Forum on Competition: Competition, State Aids and Subsidies, contribution from the U.S. Federal Trade Commission. Paris. Recuperado de http:// www.oecd.org/competition/globalforum/ GlobalForum-February2010.pdf

Organización Mundial del Comercio -[OMC]. (s.f.) Acuerdo sobre Subvenciones y Medidas Compensatorias. Recuperado de https://www.wto.org/spanish/docs_s/ legal_s/24-scm.pdf

Pérez, B. (2008). Ayudas de Estado en la jurisprudencia comunitaria. Valencia: Tirant Lo Blanch.

Pérez, J. (2012). La aplicación del Derecho de la Unión Europea sobre ayudas estatales por los Tribunales Nacionales. Revista de Derecho Comunitario Europeo, 42, 477-517.

Querub, D. (2002). Las ayudas públicas: el caso de la construcción naval. En L. Ortiz \& A. Pascual (Coords.), Derecho de la Competencia Europeo y Español (pp. 385-408). Madrid: Dykinson.

Resolución 54693 de 2013. Por la cual se imponen unas sanciones. Superintendencia de Industria y Comercio, septiembre de 2013. Recuperado de http://www.sic.gov. co/sites/default/files/estados/RELATORIA RESOLUCION_54693_DE_16_DE_ SEPTIEMBRE_DE_2013_NULE_BIENESTARINA.pdf

Resolución 54695 de 2013. Por la cual se imponen unas sanciones. Superintendencia de Industria y Comercio, septiembre de 2013.Recuperado de http://www.sic.gov.
co/sites/default/files/estados/RELATORIA_RESOLUCION_54695_DE_16 DE_SEPTIEMBRE_DE_2013_NULE_ HOGARES.pdf

Resolución 68967 de 2013. Por la cual se resuelven unos recursos de reposición. Superintendencia de Industria y Comercio, noviembre de 2013. Recuperado de http:// www.sic.gov.co/recursos_user/documentos/normatividad/Publicaciones_Despacho_3/sicdecisiones2/RESOLÜCIONES_COMPETENCIA_ANOS/2013/ RESOLUCION_68967_DE_25_DE_ NOVIEMBRE_DE_2013 RECURSO_ REPOSICION_NULE_HOGGARES.pdf

Resolución 68972 de 2013. Por la cual se resuelven unos recursos de reposición. Superintendencia de Industria y Comercio, noviembre de 2013.Recuperado de http:// www.sic.gov.co/recursos_user/documentos/normatividad/Publicaciones_Despacho_3/sicdecisiones2/RESOLUCIONES_COMPETENCIA_ANOS/2013/ RESOLUCION_68972_DE_25_DE NOVIEMBRE DE $201 \overline{3}$ RECURSO REPOSICION_NULEE_BIENESTARINA.pdf

Rodríguez, J. (2002). La participación en el capital social como modalidad de ayuda pública a las empresas. Santiago de Compostela: Escola Galega de Administración Pública.

Rodríguez, W. (2002). Las ayudas públicas en la Ley de Defensa de la Competencia. En J. Beneyto (Dir.) \& J. Maillo (Coord.), El nuevo Derecho comunitario y español de la Competencia: descentralización, análisis económico y cooperación internacional (pp. 354-408). Madrid: Bosch.

Schinkel,M.(2007).EffectiveCartelEnforcement in Europe. World Competition: Law and Economics Review, 30, 539-572. 
Schon, W. (1999). Taxation and State Aid Law in the European Union. Common Market Law Review, 36(4), 911-936.

Soto, J. (2014a). La Defensa de la competencia y el alcance de las ayudas públicas en el marco de los Tratados de Libre Comercio. Vniversitas, 128, 315-355.

Soto, J. (2014b). "Public enforcement" $y$ descentralización en la aplicación de las normas de libre competencia en la Comunidad Europea yen España. Bogotá: Universidad Externado de Colombia.

Soto, J. (2014c). La protección al consumidor como finalidad primordial de la defensa de la competencia: la experiencia de Estados Unidos, la Unión Europea y Colombia. Díkaion, 23(2), 351-421.

Tasan-Kok, T., Groetelaers, D., Haffner, M., Van der Heijden, H., \& Korthals W. (2013). Providing Cheap Land for Social Housing: Breaching the State Aid Regulations of the Single European Market? Regional Studies, 47(4), 628-642.

Tiebout, C. (1956). A pure theory of local expenditures. Journal of political economy, 64(5), 416-424.

Unión Europea. Sentencia 30/59 (1961, febrero 23). Steenkolenmijnem vs. La Alta Autoridad de la CECA, asunto. Tribunal de Justicia de la Unión Europea.

Unión Europea. Sentencia C-6/64 (1964, julio, 15). Flaminio Costa vs. Ente Nazionale Energía Elettrica, empresa que pertenecía anteriormente a la Edison Volta (ENEL), asunto. Tribunal de Justicia de la Unión Europea. Unión Europea.

Unión Europea, Sentencia C-278/93 y otros (1994). España/Comisión, asunto. Tribunal de Justicia de la Unión Europea, Unión Europea.

Unión Europea. Sentencia C-39/94 (1996, julio 11). Syndicat français de l'Express international (SFEI) y otros vs. La Poste y otros, asunto. Tribunal de Justicia de la Unión Europea. Unión Europea.

Unión Europea. Sentencia del Tribunal de Primera Instancia de la Unión Europea T-14/96.6 (1999). Bretagne Angleterre Irlande vs. La Comisión de las Comunidades Europeas, asunto. Tribunal de Primera Instancia de la Unión Europea. Unión Europea.

Unión Europea. Sentencia C-256/97 (1999, junio 29). Déménagements-Manutention Transport $S A$, asunto. Tribunal de Justicia de la Unión Europea. Unión Europea.

Unión Europea. Sentencia C-482/99 (2002, mayo 16). República Francesa vs. la Comisión de las Comunidades Europeas, asunto. Tribunal de Justicia de la Unión Europea. Unión Europea.

Unión Europea. Sentencia N 02-04641 (2003, junio 24). Societé Brit Air vs. Chambre de Commerce et díndustrie de Strasbourg et du bas-rhin Societe Ryanair, asunto. Tribunal Administrativo de Estrasburgo. Unión Europea.

Unión Europea, Sentencia C-232/05 (2006, octubre 5). Kimberly Clark, asunto. Tribunal de Primera Instancia de la Unión Europea. Unión Europea.

Unión Europea. (2007). Tratado de Funcionamiento de la Unión Europea. Diario Oficial No. C 326/47.

Unión Europea. (2010). Tratado de Funcionamiento de la Unión Europea. Diario Oficial No. C 83/47. Unión Europea. 
Unión Europea. Sentencia C-288/11 P (2012, diciembre 19). Mitteldeutsche Flughafen AG y Flughafen Leipzig-Halle $\mathrm{GmbH}$ Vs. La Comisión de las Comunidades Europeas, asunto. Tribunal de Justicia de la Unión Europea. Unión Europea.

Urrea, M. (2007). Ayudas de Estado y capacidad fiscal de los entes subestatales: la doctrina del caso Azores y su aplicación a las Haciendas Forales Vascas (Comentario a la STJCE de 06/09/06, República de Portugal/ Comisión, As. C-88/03). Revista Electrónica de Estudios Internacionales, 14, 1-24.
Valdés, L. (1992). Ajuste estructural de la rama construcción naval en la C.E.E. (19731988). Oviedo: Universidad de Oviedo.

Whish, R., \& Bailey, D. (2012). Competition Law. Oxford: Oxford University Press.

Wood, D. (2007). Cuno vs. Daimler Chrysler, Inc.: State Aids from an American Perspective. European State Aid Law, 6(1), 8. doi: https://doi.org/10.21552/ESTAL/2007/1/93 